\title{
Split allocation model of intersection group oriented to network carrying capacity limit
}

\author{
Jianwei Hü ${ }^{1,2, a}$, Ren Zhang ${ }^{1,2}, \mathrm{Ya} \mathrm{Li}^{1,2}$ \\ ${ }^{1}$ Traffic Management Research Institute of the Ministry of Public Security, Wuxi 214151, China \\ ${ }^{2}$ Wuxi Huatong Intelligent Transportation Technology Development Co., Ltd., Wuxi 214122, China
}

\begin{abstract}
In view of the network carrying capacity limit under over-saturated condition, a split allocation model of intersection group was developed, which takes total network delay, total output and total queue length as a comprehensive evaluation index. Based on the analysis of the influence of traffic flow turning, road section capacity, signal cycle length and split on traffic control, under the condition of network carrying capacity limit, the global optimization of intersection group splits was realized, taking the network traffic operation state as the basic judgment criterion and the corresponding constraint conditions were constructed. The numerical example analysis and simulation results show that the proposed model can ensure the traffic signal control effect under the premise of different demand states and control objectives, so that the effective prevention and quick dredging of traffic congestion can be realized under the condition of the network carrying capacity limit.
\end{abstract}

\section{Introduction}

The traffic congestion at the network level means that the key sections and intersections that play the supporting role of the network skeleton reach the limit of traffic capacity. Under the condition of network carrying capacity limit, the coupling correlation between signalized intersections which can directly intervene and regulate is strengthened, and the adjustment of single intersection signal control scheme is bound to have an impact on its adjacent intersections, while the traditional traffic signal control based on point or line level is limited to the improvement of local traffic conditions, which can not fundamentally solve the traffic congestion problem.

Ernesto [1] and Girianna [2] developed a global optimization model of the split and a regional coordinated control model, respectively. Daganzo [3] proposed an optimal design method of over-saturated coordinated control based on CTM. K. Aboudolas [4-5] proposed a real-time regional signal control strategy based on rolling rule quadratic planning for the congested traffic state of urban large-scale road network. The literature [6] introduced the network analysis method to optimize the traffic system control structure, in view of the lack of the inherent characteristics of the traffic network structure in SCOOT and SCATS control systems.

However, the practical significance of the split optimization for each phase of the intersection under the condition of the network carrying capacity limit is neglected, which is not conducive to reflect the positive impact of network optimization control on alleviating urban traffic congestion. Therefore, how to ensure the smooth operation of traffic under the limit value of network carrying capacity through the reasonable

\footnotetext{
${ }^{a}$ Corresponding author: hjwtime@ $163 . c o m$
} 
allocation of phase split at each signalized intersection of the network is the key to signal control of traffic network.

\section{Basic assumptions}

The over-saturated state is a subcategory in the blocking state[7], the performance is that the queue extends to the upstream intersection and has a negative impact on the upstream intersection. Generally speaking, the ultra-long queue spreads to the upstream intersection, which is impossible to dissipate in a single cycle of the intersection, and even causes the deadlock of the intersection. Based on the above research conclusions, the basic assumptions of the model are proposed as follows:

(1) The effective green time of any approach of over-saturated intersection group can be used effectively, and there is no phenomenon of empty green time.

(2) The function matching of intersection approach and exit lane is reasonable, and there is no intertwined phenomenon of traffic flow in and out.

(3) The influence of intersection approach widening and traffic flow reorganization are not taken into account.

(4) The propagation time of the starting wave at the start of green time is not taken into account.

\section{Split allocation model}

Define an over-saturated intersection group control network $G=(P, A)$, Where $P$ is the set of network nodes and $A$ is the set of road sections. $I$ and $J$ are the lane collection of each approach at upstream and downstream intersections, respectively. $S_{\mathbf{u} i}$ is the saturated flow of lane $i$ at upstream intersection, $S_{\mathbf{D} j}$ is the saturated flow of lane $j$ at downstream intersection. $\lambda_{i}(k)$ and $\lambda_{j}(k)$ are the split of the corresponding approach at upstream and downstream intersection in the $k$ th signal cycle period, respectively. $q_{\mathbf{D} j}(k)$ and $\tilde{q}_{\mathbf{D} j}(k)$ are the average arrival and outgoing traffic of lane $j$ at downstream intersection in the $k$ th signal cycle period, respectively. $U_{\mathbf{D} j}(k), \bar{U}_{\mathbf{D} j}(k)$, and $B_{\mathbf{D} j}(k)$ are the number of vehicles incoming, outgoing and stranded in lane $j$ at the downstream intersection in the $k$ th signal cycle period, respectively. $C(k)$ is the $k$ th signal cycle length.

\subsection{Model variable relationship and constraints}

\section{1) Incoming and outgoing traffic at the approach}

According to the basic assumptions of the model (1), the intersection in the control area is in an over-saturated state at this time, vehicles at each approach is driven out at a saturation flow rate in the release state. Therefore, the average incoming and outgoing traffic of each approach at the upstream and downstream intersections in the control area shall meet the following relationship:

$$
\left\{\begin{array}{c}
q_{\mathrm{D} j}(k)=\sum_{i=1}^{I} S_{\mathrm{U} i} \cdot \lambda_{i}(k) \cdot \delta_{(i, j)} \\
\delta_{(i, j)}=\left\{\begin{array}{c}
1, \quad \text { The traffic flow direction is from the upstream } \\
\text { approach } i \text { to the downstream approach } j \\
0, \quad \text { or else } .
\end{array}\right. \\
\tilde{q}_{\mathbf{D} j}(k)=S_{\mathbf{D} i} \cdot \lambda_{j}(k)
\end{array}\right.
$$

\section{2) Stranded traffic at the approach}

As can be seen from the analysis, the stranded traffic at the end of a certain cycle of the signalized intersection is equal to the sum of the stranded traffic in the previous cycle and the difference of incoming and outgoing traffic in the current cycle, the lower limit of the stranded traffic is 0 . Thus, the relationship of the stranded traffic at the approach can be obtained as follows:

$$
B_{\mathrm{D} j}(k)=\max \left\{0, B_{\mathrm{D} j}(k-1)+\left[q_{\mathrm{D} j}(k)-\widetilde{q}_{\mathrm{D} j}(k)\right] \cdot C(k)\right\}
$$

\section{3) Road section capacity constraints}

In order to maintain the dynamic balance of incoming and outgoing traffic between any node in the network for a certain period of time, the capacity constraint relationship of lane in the network is as follows:

$$
B_{\mathrm{D} j}(k) \cdot l_{v e h} \cdot \varphi_{j} \leq l_{\mathrm{D} j}, \quad \forall l_{\mathrm{D} j} \in A
$$

where $l_{v e h}$ is the average space headway of the queuing traffic; $\varphi_{j}$ is the corresponding road section capacity reduction factor $\left(0<\alpha_{j}<1\right)$, the value depends on the importance of the road section; $l_{\mathbf{D} j}$ is the distance between corresponding intersections.

\section{4) Cycle constraints}

In order to ensure the overall control effect of over-saturated intersection group and take into account the control benefit of each network node, according to the actual operation condition of intersection, the signal cycle length shall meet a certain range of values, that is:

$$
C_{p \min }(k) \leq C_{p}(k) \leq C_{p \max }(k), \quad \forall p \in P
$$


In this formula, $C_{p \max }(k)$ and $C_{p \text { min }}(k)$ are the upper and lower limits of the $k$ th signal cycle length of node $p$, respectively.

\section{5) Split constraints}

In order to ensure the safety of vehicles and pedestrians at intersections, it is necessary to set the minimum green time for each intersection, that is the split of each approach of the intersection should be greater than or equal to its minimum split, and the sum of the split of each phase of the intersection should meet certain upper limit value requirements, and the constraint relationship is as follows:

$$
\left\{\begin{array}{l}
\sum_{j=1}^{J^{*}} \lambda_{j}(k)=1-\lambda_{p l}(k) \quad\left(J^{*} \in p(j)\right) \\
\lambda_{j \min }(k) \leq \lambda_{j}(k) \leq \lambda_{j \max }(k)
\end{array}\right.
$$

In this formula, $J^{*}$ is the set of key traffic lane groups of node $p ; \lambda_{p l}(k) \quad$ is the loss of split of node $p$; $\lambda_{j \max }(k)$ and $\lambda_{j \min }(k)$ are the upper and lower limits of the $k$ th split of node $p$, respectively.

\subsection{Criteria for judging the state of network traffic}

Under the condition of the network carrying capacity limit, the traffic operation states of each node and road section in the network are mainly divided into three types: dynamic diffusion state, dynamic equilibrium state and dynamic dissipation state, the traffic flow contained in the network is also related to these three states. When the network state changes from the dynamic diffusion state to the dynamic dissipation state, the total input of the network traffic flow is less than the total output, the total network queuing traffic is decreased gradually. Therefore, the dynamic variation of network carrying traffic and total queue vehicle length can be selected as the evaluation index to judge the operation state of the network traffic.

Define $Q(K)$ and $\bar{Q}(K)$ are the total input and output traffic flow accumulated for $K$ signal cycles of the network, respectively. $E$ and $\bar{E}$ are the collection of traffic lane groups for traffic flow input and output at the boundary of the network, respectively. $L(K)$ and $D(K)$ are the total queue length and total vehicle delay accumulated for $K$ signal cycles in the network, respectively. According to the basic relation (2), the number of incoming and outgoing vehicles of lane $j$ at the downstream intersection in the $k$ th signal cycle period can be calculated as follows:

$$
\begin{aligned}
& \int U_{\mathrm{D} j}(k)=q_{\mathrm{D} j}(k) \cdot C(k)=C(k) \cdot \sum_{i=1}^{I} S_{\mathrm{U} i} \cdot \lambda_{i}(k) \cdot \delta_{(i, j)} \\
& \delta_{(i, j)}=\left\{\begin{array}{l}
1, \quad \text { The traffic flow direction is from the upstream } \\
\quad \text { approach } i \text { to the downstream approach } j ; \\
0, \quad \text { or else. }
\end{array}\right. \\
& \bar{U}_{\mathbf{D} j}(k)=\widetilde{q}_{\mathbf{D} j}(k) \cdot C(k)=S_{\mathbf{D} j} \cdot \lambda_{j}(k) \cdot C(k)
\end{aligned}
$$

The total network input traffic:

$$
Q(K)=\sum_{p \in G} \sum_{k=1}^{K} \sum_{j \in E} U_{\mathrm{D} j}(k)
$$

The total network output traffic:

$$
\bar{Q}(K)=\sum_{p \in G} \sum_{k=1}^{K} \sum_{j \in \bar{E}} \bar{U}_{\mathrm{D} j}(k)
$$

The total network queue length

$$
L(K)=l_{\text {veh }} \cdot \sum_{p \in G} \sum_{k=1}^{K} \sum_{j=1}^{J} B_{\mathrm{D} j}(k)
$$

1) Criteria for judging the dynamic diffusion state:

$$
\left\{\begin{array}{l}
L(K)-L(K-1)-[L(K-1)-L(K-2)]>0, \quad K \geq 2 \\
Q(K)-Q(K-1)-[\bar{Q}(K)-\bar{Q}(K-1)]>0, \quad K \geq 1
\end{array}\right.
$$

where $L(0), Q(0)$ and $\bar{Q}(0)$ are the initial state of the network.

2) Criteria for judging the dynamic equilibrium state:

$$
\left\{\begin{array}{l}
L(K)-L(K-1)-[L(K-1)-L(K-2)]=0, \quad K \geq 2 \\
Q(K)-Q(K-1)-[\bar{Q}(K)-\bar{Q}(K-1)]=0, \quad K \geq 1
\end{array}\right.
$$

where $L(0), Q(0)$ and $\bar{Q}(0)$ are the initial state of the network.

3) Criteria for judging the dynamic dissipation state:

$$
\left\{\begin{array}{l}
L(K)-L(K-1)-[L(K-1)-L(K-2)]<0, \quad K \geq 2 \\
Q(K)-Q(K-1)-[\bar{Q}(K)-\bar{Q}(K-1)]<0, \quad K \geq 1
\end{array}\right.
$$

where $L(0), Q(0)$ and $\bar{Q}(0)$ are the initial state of the network.

\subsection{Objective function}

To ensure that the split allocation of intersections in the network is reasonable, and maximize the efficiency of the network, according to the different control objectives, the total network delay, the total network output traffic and the total network queue length are selected as the comprehensive evaluation index to construct the objective function. At the same time, different weight values are given to express their importance. The expression of the objective function is as follows: 


$$
\begin{aligned}
\min Z & =\alpha \cdot D(K)+\beta \cdot L(K)-\gamma \cdot \bar{Q}(K) \\
& =\alpha \cdot \sum_{p \in G k=1} \sum_{j=1}^{K} \sum_{j=1}^{J}\left[B_{\mathbf{D} j}(k-1)+B_{\mathbf{D} j}(k)\right] \cdot C(k) / 2+\beta \cdot l_{v e h} \cdot \sum_{p \in G k=1} \sum_{j=1}^{K} \sum_{\mathbf{D}_{j}}^{J} B_{1}(k)-\gamma \cdot \sum_{p \in G k=1} \sum_{j \in \bar{E}}^{K} \sum_{\mathbf{D}_{j}}(k)
\end{aligned}
$$

In this formula, $\alpha$ is the weight coefficient for the total network delay $(0 \leq \alpha \leq 1) ; \quad \beta$ is the weight coefficient of the total network queue length $(0 \leq \beta \leq 1)$; $\gamma$ is the weight coefficient of the total network output traffic $(0 \leq \gamma \leq 1, \alpha+\beta+\gamma=1)$.

\section{Case study}

To illustrate the applicability of the proposed model, a simplified $3 \times 3$ network model has been outlined for experimental tests, as shown in Figure 1. It is assumed that the turning traffic flow of left $(L)$, through $(T)$ and right $(R)$ at each intersection satisfies the relationship: $L: T: R=5: 9: 6$; the total lanes capacity of each section is $S=4 \times 1800 \mathrm{pcu} / \mathrm{h}$. The minimum split allowed for each phase of each intersection is set to $\lambda_{\text {min }}=0.15$, to ensure the normal operation of traffic flow; the loss split of each node in the network is $\lambda_{p l}=0.05$.

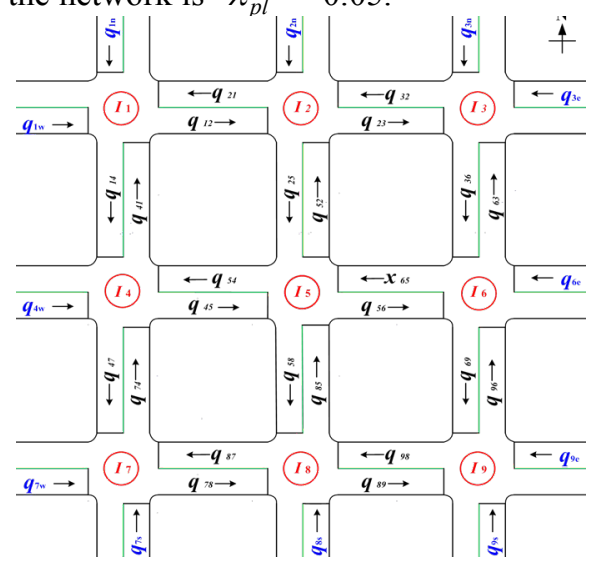

Figure 1. Network traffic flow diagram

According to the criterion of network dynamic equilibrium state and the relationship of the model, the maximum total network output traffic per unit hour is taken as the objective function, and the optimized solution is programmed with LINGO software. The maximum total network output traffic per unit hour of the network is calculated as $\bar{Q}_{\mathrm{T}}=20719 \mathrm{pcu} / \mathrm{h}$, the split allocation of each intersection and traffic flow allocation of each directed section in the network satisfying with the maximum output traffic $\bar{Q}_{\mathbf{T}}$ are shown in Table 1 and Table 2, respectively.
Table 1. Split allocation of each approach direction in the network Intersection Eastbound Southbound Westbound Northbound

\begin{tabular}{lllll}
$I_{1}$ & 0.25 & 0.23 & 0.15 & 0.32 \\
$I_{2}$ & 0.25 & 0.24 & 0.22 & 0.24 \\
$I_{3}$ & 0.33 & 0.24 & 0.23 & 0.15 \\
$I_{4}$ & 0.24 & 0.22 & 0.24 & 0.25 \\
$I_{5}$ & 0.24 & 0.24 & 0.23 & 0.24 \\
$I_{6}$ & 0.27 & 0.24 & 0.22 & 0.22 \\
$I_{7}$ & 0.24 & 0.15 & 0.31 & 0.25 \\
$I_{8}$ & 0.26 & 0.20 & 0.25 & 0.24 \\
$I_{9}$ & 0.33 & 0.15 & 0.23 & 0.24 \\
\hline
\end{tabular}

Table 2. Traffic flow allocation of each road section in the network

\begin{tabular}{cc|cc}
\hline \multicolumn{2}{c|}{ Road section Traffic flow(pcu/h) } & \multicolumn{3}{|c}{ Road section Traffic flow(pcu/h) } \\
\hline$q_{12} / q_{21}$ & $1566 / 1770$ & $q_{23} / q_{32}$ & $1652 / 1824$ \\
$q_{36} / q_{63}$ & $1573 / 1738$ & $q_{69} / q_{96}$ & $1705 / 1619$ \\
$q_{98} / q_{89}$ & $1850 / 1679$ & $q_{87} / q_{78}$ & $1719 / 1789$ \\
$q_{74} / q_{47}$ & $1569 / 1768$ & $q_{41} / q_{14}$ & $1656 / 1818$ \\
$q_{25} / q_{52}$ & $1711 / 1704$ & $q_{58} / q_{85}$ & $1720 / 1668$ \\
$q_{45} / q_{54}$ & $1705 / 1720$ & $q_{56} / q_{65}$ & $1696 / 1757$ \\
\hline
\end{tabular}

Under the condition of dynamic equilibrium, the relationship between the actual average traffic flow of the network optimized by the model and the capacity of each approach at the intersection is compared, as shown in Figure 2.

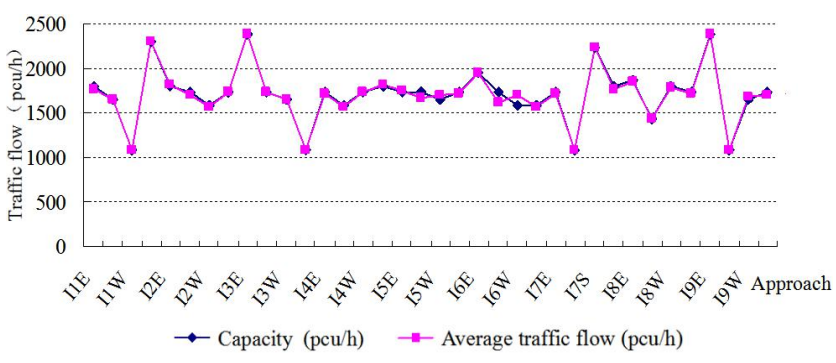

Figure 2. Relationship between actual average traffic flow and capacity after optimization

It can be seen from Figure 2 that under the condition of judging the dynamic equilibrium state of the network, the actual average traffic flow of the network optimized by the model is similar to that of each road section, and the network traffic is in a dynamic equilibrium state. At this time, the split allocation of the intersection group in the network is reasonable, which can not only ensure that the network traffic is in a stable and orderly operation state, but also realize the maximization of the total network traffic output. 


\section{Simulation experiment}

Based on the given intersection geometric conditions and traffic flow input parameters, the simulation road network is established in VISSIM 4.3 environment, and the total network output vehicle number per unit hour is selected as the final simulation evaluation output index. The output traffic volume per hour and the deviation rate relative to the theoretical value are simulated in any random phase sequence group, as shown in Table 3

Table 3. Comparison experiment of VISSIM simulation

\begin{tabular}{|c|c|c|c|c|c|}
\hline Random signal phase sequence & Phase sequence 1 & Phase sequence 2 & Phase sequence 3 & Phase sequence 4 & Phase sequence 5 \\
\hline Experiment value $(\mathrm{pcu} / \mathrm{h})$ & 20813 & 20742 & 20339 & 20284 & 20327 \\
\hline Deviation rate (relative to & $+0.45 \%$ & $+0.11 \%$ & $-1.83 \%$ & $-2.10 \%$ & $-1.89 \%$ \\
\hline
\end{tabular}

It can be seen from Table 3 that the calculation results of split allocation model of intersection group oriented to network carrying capacity limit proposed in this paper are similar to those of simulation experiments, and the deviation rates are all less than 3\%. Thus, the accuracy and reliability of the model are verified, and the output traffic maximization of the network during the peak period of the over-saturated intersection group is effectively guaranteed.

\section{Conclusions}

In this paper, a split allocation model of intersection group oriented to the network carrying capacity limit is constructed, and the signal control of traffic network under the premise of different traffic demand states and control objectives is optimized. Compared with the existing models and algorithms, the model proposed in this paper can select the corresponding optimal control criteria according to the difference of the control objectives and the network traffic demand. Through optimizing the signal cycle length and split of over-saturated intersection group, the effective prevention and quick dredging of network traffic congestion can be realized, and finally the utilization benefit of network time-space resources can be maximized.

\section{Acknowledgments}

This research is supported by National Engineering Laboratory Funded Project "Road network bearing capacity and operation reliability evaluation methods based on critical path analysis" (Project No. 2019SJGC01).

\section{References}

1. Daganzo C F. The cell transmission model, part II: Network traffic. Transportation Research Part B, 29(2):79-93, (1995).

2. Girianna M, Benekohal R F. Using genetic alorithms to design signal coordination for over-saturated networks. Journal of Intelligent Transporation Systems: Technology Planning and Operations, 8(2): 117-129, (2004).

3. K. Aboudolas, M. Papageorgiou, E. Kosmatopoulos. Store-and-forward based methods for the signal control problem in large-scale congested urban road networks. Transportation Research Part C, 163-174, (2009).

4. K. Aboudolas, M. Papageorgiou, A. Kouvelas, et al. A rolling-horizon quadratic-programming approach to the signal control problem in large-scale congested urban road networks. Transportation Research Part C, 680-694, (2010).

5. Ernesto C, Gaetano F. Combined signal setting design and traffic assignment problem. European Journal of Operation Research, 155(3): 569-583, (2004).

6. Wang L, Liu X M, Wang J Z, et al. Urban traffic signal system control structure optimization based on network analysis. Mathematical Problems in Engineering, 2013: 1-9, (2013).

7. Pignataro L J, Mcshane W R, Crowley K W, et al. Traffic control in over saturated street networks. Transportation Research Board, (1978) 